\title{
XLIV. An examination of the question, whether the discordancy between the characteristics of mechanical electricity, and the Galvanic or Voltaic Fluid, can arise from difference of intensity and quantity; with some observations in favour of the existence of an electro-motive power independently of chemical reaction, but cooperating therewith: respectfully submitted to the British Association for the advancement of Science
}

\section{R. Hare M.D.}

To cite this article: R. Hare M.D. (1836) XLIV. An examination of the question, whether the discordancy between the characteristics of mechanical electricity, and the Galvanic or Voltaic Fluid, can arise from difference of intensity and quantity; with some observations in favour of the existence of an electro-motive power independently of chemical reaction, but cooperating therewith: respectfully submitted to the British Association for the advancement of Science, Philosophical Magazine Series 3, 9:53, 212-220, DOI: 10.1080/14786443608636479

To link to this article: http://dx.doi.org/10.1080/14786443608636479 
integral, as a decided denial of my statement. In my reply I observed that a direct process of integration always leads to the singular solution at the same time with the general solution, thereby showing that the general result of the investigation does really furnish every possible solution in accordance with what I had said. Is it not, then, evident that the subject of singular solutions was utterly useless as an objection to my remark, that Professor Young had not sufficiently examined the matter, and that his expression, that I had done myself a "wanton injustice," was not justifiable on the score either of accuracy or of propriety?

I hereby close my remarks on this subject for the present. It is obvious that Professor Young, instead of applying his own mind to the discussion of the points at issue, has all along rested his evidence and opinions on a misinterpretation of authorities. I do not suppose I should have written these brief and concluding observations for the readers of the Journal, had I not felt myself imperatively called upon to notice the tone of assumption and dictation that prevails throughout his last letter. He ought not to be unconscious of the fact, that personal sarcasms and presumptuous language are not the generally received indications of a strong supply of argument or of a sincere desire after the development of truth.

London, August 13, 1836.

XLIV. An Examination of the Question, whether the Discordancy between the Characteristics of Mechanical Electricity, and the Galvanic or Voltaic Fluid, can arise from Difference of Intensity and Quantity; with some Observations in Favour of the Existence of an Electro-motive Power independently of Chemical Reaction, but cooperating therewith : respectfully submitted to the British Association for the Advancement of Science. By R. Hare, M.ID., Professor of Chemistry in the University of Pennsylvania.*

I $\mathrm{N}$ one of the papers, giving an account of Faraday's recent valuable researches in electricity, for copies of which $I$ have been indebted to the flattering attention of the author, I find the following language:

"Hence arises still further confirmation, if any were required, of the identity of common and voltaic electricity; and that the differences of intensity and quantity are quite sufficient to account for what were supposed to be their distinctive qualities †." And elsewhere referring to Cavendish, as the author of this opinion, it is alleged that it "only requires to be un-

* Reprinted from a pamphlet privately circulated by the Author.

† [See Lond. and Edinb. Phil. Mag., vol, iii. p. 363.-Eort.] 
derstood in order to be admitted." Notwithstanding that in support of the opinion thus quoted, the much-respected authority of both Cavendish and Faraday is arrayed, it is one which I cannot so understand as to admit.

I am unable to form any other idea of intensity, than that of the ratio of quantity to space. Thus the intensity of the pressure of an elastic fluid, is as the quantity to the space in which it is confined. The space being the same, the intensity of the pressure will be directly as the quantity; the quantity being constant, the pressure will be inversely as the space.

Agreeably to an analogous mode of reasoning, the intensity of the light or the heat emanating from a radiant body, is always estimated to be inversely as the surfaces on which it may be diffused or concentrated; and hence the inference that the intensity is as the square of the distance inversely, or as the area of the receiving surface of a lens or mirror, to that of the focus into which the rays are collected.

It follows that if there be in any two cases, like quantities of electricity evolved by mechanical, and by galvanic apparatus, the space occupied by the fluid generated by the latter, must be as much greater, as its intensity is less.

In a memoir which I published upon this subject some years ago, I endeavoured to show that the spaces occupied by equivalent charges of galvanic and mechanical electricity were not such as to justify the idea that the former required for its existence a larger space than the latter. But on this subject, it is not now necessary to recur to the facts which I then adduced, since I find it conceded in one of the recent Memoirs of Faraday, that the spaces occupied by the electricity evolved by galvanic apparatus, as compared with those occupied by mechanical electricity, are almost infinitely small. " $A$ grain of roater or of zinc, contains as much of the electric fluid as zoould supply eight hundred thousand charges of a battery containing a coated surface of fifteen hundred square inches." "Four grains of zinc, with one of water, may yield as much electricity as is evolved during a thunder storm."

It follows inevitably that the electric matter evolved by galvanic action is, previous to its evolution, in a state almost infinitely intense, as compared with that of the same matter, when evolved by a machine or meteorological changes. Yet to the currents induced in this matter, in the first-mentioned form, an opposite state is ascribed, as respects intensity, to that in which it has previously existed.

It may be said with respect to currents, that the space being the same, the intensity will not only be directly as the quantity, but also inversely as the time in which it passes, or, in other 
words, directly as the velocity. But what is to create inequality of velocity when the channel, a wire for instance, is of the same size and nature in both cases? When the same fluid is in question, the velocity will be as the forces by which it may be impelled. The only forces to which electricity has ever been alleged to be liable, as far as I am informed, are either the self-attractive or self-repulsive power of its own particles, or their attraction for other matter. It will be admitted that the intensity of these forces must, in the case of electricity, as in that of caloric or light, be as the quantity to the containing space; and consequently, that it wonld be unreasonable to allege that the reciprocal repulsion of the electric atoms, or their attraction for other matter, and consequently any velocity thence arising, should not be in proportion to the state of condensation from which they may be liberated.

If the superior velocity displayed by electricity generated by friction be the cause, not the consequence of greater intensity, how are we to account for the superior velocity?

According to the doctrine of Du Fay, electricity is retained upon the surface of a charged pane by the reciprocal attraction of the heterogeneous fluids. According to the Franklinian doctrine it is retained by its attraction for the negative surface, on which side this attraction is not counterbalanced by repulsion from other electricity. When the circuit is completed by a conductor, according to the one doctrine, a surcharge on the one side is translated to the other; while, according to the other doctrine, two heterogeneous fluids rush from the surfaces in which they are previously accumulated in excess, to enter into combination, and, at the same time, to restore the equilibrium of the surfaces on which they have been respectively deficient. But according to either hypothesis, wherefore should the forces be greater for electric matter when generated by one means, than when produced by another? Why should a mass of electric matter evolved in a diffuse state by a machine, or from a cloud, rush like a bullet through conductors, which are almost impassable to the same fluid when evolved from a state of extreme density within a simple galvanic circuit?

During the process of exciting an electric battery, the electricity previously existing equally on both sides of the glass is so transferred from one side to the other, that the one be comes as much negative as the other becomes positive; and it must be evident that the intensity will be limited by the extent of the force by which this transfer is effected. It is difficult to conceive that merely by a change of capacity arising from friction, a force should be generated at all comparable to that which the electric matter must exert, in escaping, according 
to the premises, from a state of extreme density, as when extricated by galvanic action from water or zinc.

I ascertained, some years ago, that the galvanic fluid evolved by a large calorimotor of a single pair, will not ignite a wire which may be easily deflagrated by a much smaller apparatus of the same construction. Yet sheets of metal, about four inches in breadth, might be raised by a discharge from the larger instrument above the temperature of boiling water. In such cases, agreeably to the doctrine of quantity and intensity, the electric fluid exists, up to the period of its evolution, in a state of extreme condensation and consequent intensity, and yet at the moment when a perfect but restricted channel is afforded to it, becomes too diffuse to pass through it with a velocity sufficient to produce deflagration. How can the electricity which is in the one case so dense, become in the other so rare? Where, and in what manner does it exist intermediately between the period of its condensation within the pores of the generating materials, and its rarefaction in the wire which forms the circuit between them?

I am aware that to the want of adequate insulation, the inferior intensity of the charges communicated to coated surfaces by voltaic apparatus, will be attributed; as it cannot, without a palpable contradiction, be ascribed to any defect of intensity, in a source wherein the ratio of the quantity to the space is almost infinitely great.

Let us, then, examine the subject agreeably to this view of the case. Since the electricity liberated by electro-chemical reaction by means of a single galvanic pair must have preexisted in a state of extreme condensation and consequent intensity, it follows that it ought to be productive of a tension limited only by the insulating power of the menstruum within which it is extricated. It should then, when evolved as above described, attain the highest degree of intensity consistent with the insulating power alluded to. That this is not the fact, is fully established by general experience, and by the observations of Faraday, according to which the intensity of a voltaic series increases with the number of pairs employed.

It results also from the premises, that the tension should become as great in a large as in a small pair; and by employing one large pair, effects should be attainable, as potent in respect to intensity, and more potent as respects quantity, than those resulting from a series of pairs. Yet the experiments above mentioned prove, that as the surfaces, associated as a single galvanic pair, are enlarged, the intensity lessens; so that a calorimotor of a single pair containing fifty square feet of zinc, will not, in a wire of any size, produce an ignition of as high 
intensity as may be effected by the elementary battery of Wollaston, formed of a silver thimble, and piece of zinc proportionably minute.

It appears from the experiments of Professors W. B. and H. D. Rogers*, that the power of a galvanic pair in deflecting a magnetic needle, was increased by causing the surface of the copper plate to exceed that of the zinc; while by extending the zinc surface beyond that of the copper, little or no increase of power ensued. This result appears to be the opposite of that which the theory of Wollaston, supported by some recent observations of Faraday, would lead us to expect. As pursuant to that theory, the galvano-electric fluid is due exclusively to chemical reaction; if the charge were not promoted by an excess of extension in the oxidizable metal, it ought not to have been improved by similar extension of that which is insusceptible of oxidizement.

According to the observations of the Professors above mentioned, the deflection resulting from a galvanic discharge, on the first immersion of the plates, after a repose of two hours, was six times as great as that which could be permanently sustained. The greatest effect appeared always to ensue before there was any sensible extrication of hydrogen, and the commencement of the effervescence was invariably the signal for a decline of power.

It was by analogous observations respecting the igniting influence of galvanic apparatus that $I$ was led to the construction of my deflagratorst, in which the deflagrating power appears, agreeably to my experience, to be exalted as much by the repose of the surfaces as the ability to influence the magnetic needle was ascertained to be, in the experiments of my sagacious friends above mentioned.

That the evolution of the galvanic fluid is not in proportion to the intensity of the chemical reaction, is corroborated by the fact, that the intensity of the ignition, excited in a wire by a galvanic discharge, diminishes, while the effervescence increases; and it is well known that the power of galvanic apparatus is not augmented by adding to the strength of the solvent, beyond a very moderate limit.

But if it be granted that chemical affinity, when reacting within a galvanic circuit, without any propulsive power from the elements of the circuit, can receive a peculiar impulse, so as to produce a current of the electric fluid, confining it at the same time to a very narrow channel, by what process can this species of chemical reaction be conceived to accelerate an elec-

- See Silliman's Journal for October, 1834.

$\downarrow$ [See Phil. Mag., First Series, vol. lvii, p. 281, lix. p. $113 . \&$ lxiii. p. 241. -EDIT. 
trical current already produced? The same propulsion must be given to the electricity liberated between the plates of the second pair as between those of the first; and it is inconceivable to me, that the accession of that derived from the first pair should add to the velocity of the portion evolved by the second. The current of the former cannot be supposed to move with greater velocity on account of its meeting with another, which moves at its own rate. Currents are not accelerated by their confluence, unless the head or pressure be increased, and the channel restricted. But in the case in point it has been shown, that by the reaction of the solvent with the first pair in the series, the tension must attain the highest degree consistent with the imperfect insulation; and no cause has been assigned for the restriction of the channel. It may be said that the current from the first pair cannot pass through the liquid in the second cell without causing the decomposition of that liquid; and that as its power is inadequate to effect this change, it has to pursue the same route as the electricity which is generated by the oxidizement of the second plate of zinc. It is still difficult to me to imagine that it can transfer its momentum to the current which thus precedes it; or that the chemical reaction by which the latter is evolved, should act only in accelerating the stream which it receives from the preceding pair. Granting that imponderable matter, at the moment of its extrication from confinement among ponderable atoms, were to receive an impulse which, by extraneous cooperating causes, should force it to move in a current, yet I cannot imagine that such atoms can, by any reaction originating between themselves, give an impulse to imponderable matter extricated from other atoms. Whether or not the electricity be derived from chemical reaction, it seems to me that the power which puts it in motion, and accelerates and condenses it into a channel, still smaller and smaller as its intensity increases, must be ascribed to some mysterious property arising from the arrangement of the elements of the series, which is, in the present state of our knowledge, inexplicable.

This electromotive power, if not antecedent, does not appear to me to be consequent to chemical reaction. I conceive that it operates upon all the imponderable elements within its scope, tending to accumulate them at the "electrodes" under a greater or less degree of tension. The potency of the resulting discharge, when the circuit is completed, is regulated both by the tension and the quantity of the imponderable matter accumulated. But the presence of reagents, which favour the extrication of imponderable materials, as in the more efficient voltaic apparatus, is compatible only with a feeble insu-

Third Series. Vol. 9. No. 53. Sept. 1836. $2 \mathrm{~B}$ 
lation, while arrangements more favourable to insulation, as in De Luc's Electric Column, are incompatible with a copious supply of the imponderable matter.

Probably upon an analogous ability to produce or annul, to promote or retard, chemical reaction, the efficacy of animal and vegetable organization is founded, being obviously dependent on an arrangement of masses. The voltaic series of a gymnotus is evidently an animal organ, and its analogy with the voltaic series produced by human ingenuity induces me to consider the latter in the same class of agents as the organs by which life is supported.

I should have expected, that in establishing the highly interesting fact that every elementary equivalent has the same quantity of electricity, the ingenious author of this discovery would have adverted to the analogous observations of Petit and Dulong respecting the specific heat of elementary atoms. It strikes me as important, that similar conclusions should have been arrived at by such high authority, both as respects caloric and the electric fluid. I am surprised that Faraday should appear to have overlooked this analogy in the explanation of the practical results which he has obtained.

No hypothesis appears to be more generally sanctioned at this time among chemists than that which ascribes the aeriform state to a union between caloric and ponderable matter. When hydrogen unites with oxygen, caloric is evolved. It follows that when these substances are made to resume the gaseous form, caloric must be supplied to them.

When it is considered that the inferences of Petit and Dulong, respecting the specific caloric, and those of Faraday respecting the electricity combined with ponderable equivalents, tend to demonstrate the coexistence in them of equivalent atmospheres of each of those imponderable fluids, does it not authorize a surmise that in the voltaic current they may be associated; and that with those equivalent measures of electricity which Faraday has shown to pass, corresponding portions of caloric are imparted? The idea of Berzelius, "that the heat and light evolved during porverful combinations, are in consequence of an electric discharge at the same moment taking place," being cited by Faraday in the language of this quotation, he observes, that it " is strictly in accordance with his view of the quantity of electricity associated with the particles of matter." To me it appears to be no less in accordance with the idea that heat and light are associated with those atoms to a commensurate extent; and since, by the premises, electricity reacts with them, they may be presumed to react with electricity. 
That heat, light, and electricity are all concomitant products of electro-chemical reaction, is self-evident. Agreeably, then, to the strict rules of induction, wherefore is the principle last mentioned to be considered as the cause of the others?

Where is the proof that the heat and light evolved between the "electrodes" are effects merely of electricity? The fact of the apparently unlimited evolution of heat from a finite portion of wire duly subjected to a voltaic circuit, is inexplicable, consistently with the materiality of caloric, unless we suppose the fluid to be derived from the same electro-chemical reaction to which we owe the electricity associated therewith.

I conceive it to be almost self-evident, that mechanical and voltaic electricity are due to the same fluid, so far as they are strictly electrical. The only doubt with me is, whether the very different characteristics of the phænomena produced by the different means alluded to could be explained without supposing some other modifying causes. And at all events, from the reasons above given, I am dissatisfied with the explanation that the difference is dependent on quantity and intensity.

In terminating my observations, I subjoin the following statement of my opinions as heretofore expressed in one of $\mathrm{my}$ text-books.

"It does not appear to me that the production of electromagnetic phænomena, both by galvanic and by electrical discharges, disproves my opinion, that caloric and electricity are connate and coordinate products of galvanic action.

As ignition is producible by either discharge, whether electric or galvanic, the fluid of heat, no less than the electric fluid, may in both cases be concerned; and it is yet to be shown, that magnetic phænomena are ever due to the unalloyed agency of electricity.

It is true that magnetism has been imparted, by discharges of mechanical electricity, without any ostensible agency of caloric; but it is equally true, that magnetic movements have been produced also, by the application of heat, unaccompanied by any ostensible agency of the electric fluid; and it seems as rational to suppose that caloric and electricity are associated in the first instance as in the last.

Those who consider electricity, varying in quantity and intensity, as the common cause of electrical and galvanic ignition, and of thermo-magnetic phænomena, must suppose that this principle and caloric are capable of a reciprocal action. In the first case, caloric is evolved by electric action ; in the last, electric currents are produced by calorific repulsion. Hence, as action and reaction are equal and contrary, I deem it rational to suppose that in some cases the former, in other cases 2 B 2 
the latter, may be the prime mover; but that both participate in every galvanic, electro-magnetic, or thermo-magnetic current.

XLV. On the Difference between the Attractive Porvers for soft Iron of the Electro-magnet and the Steel Magnet; in reply to Dr. Ritchie. By G. RaIney, Member of the Royal College of Surgeons.

To the Editors of the Philosophical Magazine and Journal.

\section{Gentlemen,}

Tr HE last Number of the Philosophical Magazine, at page 83, contains some remarks by Dr. Ritchie upon a paper which I communicated in May last (printed in the Number for July), concerning the cause of the great disproportion in the attractive power of the electro-magnet for soft iron when in contact and at a distance, compared with the attractive power of the common steel magnet under similar circumstances. The concluding paragraph of Dr. Ritchie's paper is as follows :

"In the explanation given by Mr. Rainey, the lifter is supposed to react powerfully on the electro-magnet, so as to increase its power, a supposition which cannot possribly be admitted. For the electro-magnet must, in the first place, give the lifter all its magnetic power, consequently the power of the lifter can never exceed that of the electro-magnet, and consequently never can induce a higher magnetic state in it than what has already been done by the voltaic helix." What Dr. Ritchie pronounces to be a supposition which cannot possibly be admitted, I will prove, I think to Dr. Ritchie's satisfaction, both by reasoning and experiment, to be an absolute and undeniable fact. My position is, that the lifter of a common horse-shoe magnet can exceed the power of the magnet to which it is applied; and in case that magnet has not received its maximum of magnetism, can induce in it a higher state of magnetism than that which existed before the application of the lifter; and that in proportion as the permanent magnet resembles in its texture soft iron, the effects of induction on it and on the electro-magnet more closely resemble each other, so as to render it obvious that the effect of the keeper on the two is the same, excepting in degree.

Let $a$ and $b$ denote the inducing power of the magnet $\mathrm{A}$, and let $B$ be a piece of soft iron, placed in the position represented in the annexed diagram, and sufficiently large to receive all 